\title{
A New Mathematical Model Concept and Challenges in relation to the Layout of Cluster Manifolds
}

\author{
Conglei Chen ${ }^{1, *}$, Yang $\mathrm{Du}^{2}$, Hui Huang ${ }^{1}$, Yu Zhao ${ }^{2}$, Yingying Wang ${ }^{2}$ and Menglan Duan ${ }^{2}$ \\ ${ }^{1}$ Petroleum Exploration \&Production Research Institute, SINOPEC, Beijing, China, 100083 \\ ${ }^{2}$ Institute for Ocean Engineering, China University of Petroleum, Beijing, China, 102249 \\ ${ }^{*}$ Corresponding author
}

\begin{abstract}
The cluster manifold becomes an essential part in a subsea production system, which can gather the production fluid from subsea wells and distribute water, gas and chemical agents from the floating production system to each subsea well. On account of its advantages such as a small initial investment, being installed before arrival of floating platforms, and optimizing the layout of the subsea production system, the manifold system has been widely used in the development of ultra-deepwater oil and gas fields. But many factors affect the layout design of cluster manifold. In addition, due to the particularity of deepwater target reservoir, uncertainty of project construction, and oil company short and long term marketing strategy etc., the engineers' rich experiences are highly demanded in the layout of cluster manifolds. In this paper, a new mathematical model concept is presented and the mathematical models for the layout of cluster manifold are roughly proposed. Meanwhile, the challenges in relation to the partition problem of subsea wells and the connection paths between subsea facilities are analyzed. The optimization layout of cluster manifold using the mathematical model is an exploratory attempt, which can provide a quantized reference to the engineers for the evaluation of the optimal scenario.
\end{abstract}

Keywords-subsea production system; cluster manifolds; optimization concept; challenges

\section{INTRODUCTION}

Currently, there are mainly four layout types in the subsea production system, which are single well tie-backs, daisy chains, template manifolds and cluster manifolds (Mclnturff, 1998 and Stock et al, 2007). Each layout type has its own suitable application background due to the characteristics of deepwater target oil and gas fields. Compared to the other three layouts, the cluster manifold as the hub manifold of subsea production system plays an important role in providing a collection and distribution point for production fluid and water, gas and chemical agents injection. In the layout of cluster manifolds (See Fig.1), there are many advantages such as small initial capital expenditure (CAPEX), pre-installation, short time for the first oil, flexible scalability and so on. Therefore, the cluster manifold becomes more and more popular and the engineers prefer it in spite of several disadvantages such as the more work in connection and adjustment along with the higher operating expense (OPEX) in maintenance during the operating phase.

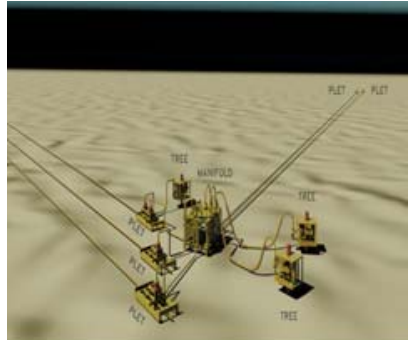

(a) One cluster manifold

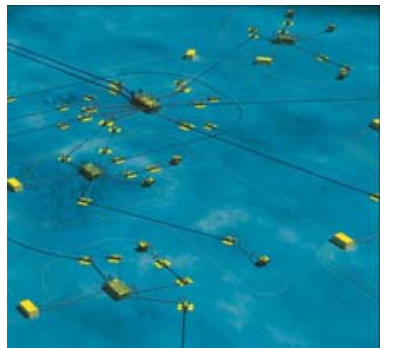

(b) Many cluster manifolds
FIGURE I. THE LAYOUT OF CLUSTER MANIFOLDS

The layout of cluster manifolds is a key problem in wet wellhead development scenario, which has a significant impact on the evaluation of the development scenario, investment and income, equipment reliability, and integrity management of deepwater facilities. Therefore, it is necessary to optimize the number and locations of cluster manifolds, connection relations between subsea facilities, and the design of pigging loop. Nowadays, the determinations of final layout scenario of cluster manifolds, connection and adjustment quantities, risk and cost mainly depend on many factors, which means the engineer's experience more important. But it is not always effiecient because of the high uncertainties of factors, which makes that a lot of manpower and engineering resources are demanded. To provide a quantitative reference and assist the engineers in making their decision, it is necessary to develop some software tools using mathematical models.

In fact, some scholars did deepwater technical research with related mathematical models to reduce CAPEX and OPEX. Kaiser and Allan (2007) researched the generalized functional models for drilling cost estimation. Onwunalu and Durlofsky (2009) presented the development and application of a new well pattern optimization algorithm for optimizing large-scale field development. Ciaurri et al. (2012) studied the integrating mathematical optimization and decision making in intelligent fields. Wang et al. (2012) proposed a mathematical model for the partition of subsea wells in the layout of cluster manifold and developed a dedicated algorithm to solve this optimization problem. Although some people have focused on the layout of cluster manifold by mathematical modeling, many efforts need to be made to research the problem of inherent complexities.

In this paper, based on the previous work, a new concept of the optimization layout of cluster manifold by the mathematical means is presented. Meanwhile, aiming to the difficulties of partition of subsea wells and optimal connection paths among 
cluster manifold, floating production platform and PLEM, three mathematical models are proposed and some challenges in the models are anlyzed, which will provide a good reference for future study.

\section{Two Main Problems in the Layout of Cluster MANIFOLD}

\section{A. Partition of Subsea Wells}

Well slots on each cluster manifold are used to connect to subsea trees by connection facilities. The number of well slots is the maximum number of subsea wells which can be connected to the cluster manifold. There are many kinds of cluster manifolds with different number of well slots, such as 2-slots, 4-slots, 8-slots and so on (Wang et al, 2012). If the number of well slots is greater, the weight and size of cluster manifolds are larger. As a result, the installation of cluster manifold becomes more difficult because of deepwater resources scarcity and many challenges. In general, the cluster manifold with 4-slots, 6-slots or 8-slots is widely used in deepwater engineering.

If there are many subsea wells in a large-scale oil and gas field, all the subsea wells to be connected to one cluster manifold with greater size is not suitable and more cluster manifold with smaller size may be demanded. When the number and locations of subsea well are known, the optimal number and locations of cluster manifolds should be determined. In addition, connection relations between cluster manifolds and subsea wells need to be optimized to decide whether the subsea wells can be connected to the same cluster manifold. Fig. 2 shows the partition of subsea wells, where there are 10 subsea wells.

If two 6-slots cluster manifolds are used, possible partition is shown in Fig. 2(a), where 2 well slots can be left for the future tie backs of subsea wells. If three 4-slots cluster manifolds are used, possible partition is shown in Fig. 2(b), where 2 well slots can be also left for the tie back. It is noted that a subsea well can be only connected to one of the cluster manifolds. If the different number and types of cluster manifolds are adopted, different layout scenarios can be gotten. Thus, the optimal partition needs to be found based on many factors.

Therefore, how to partition subsea wells into each cluster manifold and obtain the optimal number and locations of the cluster manifolds with the minimum layout cost and risk, which is an important issue.
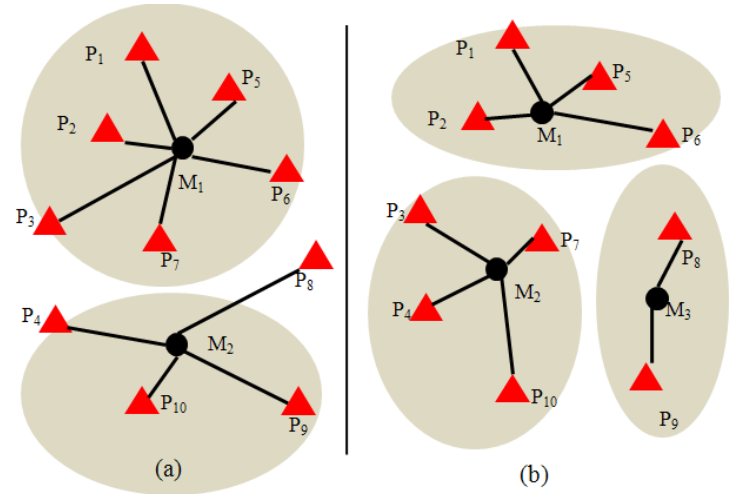

FIGURE II. DIFFERENT PARTITION OF SUBSEA WELLS

\section{B. Connection Paths}

If there is one cluster manifold, the production fluid will be lifted to the floating production platform by the risers and the export pipelines from the headers on the cluster manifold. But if there are two or more cluster manifolds, the connection paths between clusters manifolds need to be discussed for the transportation of the production fluid. In engineering, there are two main connection types between cluster manifolds, i.e., in series and connection by means of the pipeline end manifold (PLEM).

If the connection types between cluster manifolds are in series, there is an optimal connection path to connect different cluster manifolds one by one by connection facilities and the export pipelines are connected with the headers on the cluster manifold closer to the floating production platform to transport the production fluid. When there are many cluster manifolds, the series connection may make the diameter of export pipeline much larger and the length of flowlines much longer, which may induce a great challenge for flow assurance and installation of flowlines. It is better to use the PLEM to gather all the export pipelines from the headers on cluster manifolds and just two headers on the PLEM are used to transport the production fluid, which can decrease the number of pipelines and risers, cost and risk.

It is noted that the connection types between cluster manifolds may exist alone or two mixed forms. How to obtain the optimal connection paths and types needs to be discussed in the optimization layout of cluster manifolds.

\section{SOME STATEMENTS}

To understand the problem better, some statements are made as follows:

(1) Floating production storage and offloading (FPSO) is used to represent the floating production platform, whose coordinate (mapping position from subsea surface) is taken as the location of the riser.

(2) The locations of subsea facilities and FPSO are in a two-dimensional coordinate system.

(3) There is no limitation of submarine topography and some policy regulations, i.e., subsea facilities are allowed to be installed everywhere in the seabed. 
(4) In the mathematical models, the lowest layout cost (CAPEX) is our target function and proportional to the length of pipelines. The evaluation of risk is not included.

\section{Difficulties AND Challenges IN THE MATHEMATICAL MODELS}

We assume that the optimal layout scenario of cluster manifold can be obtained with one FPSO, $n$ subsea wells $p_{1}, p_{2}, \cdots, p_{n}, m$ cluster manifolds $M_{1}, M_{2}, \cdots M_{m}$, and $l$ PLEMs $P M_{1}, P M_{2}, \cdots P M_{l}$. The problem is how to partition $n$ subsea wells into $m$ cluster manifolds and get the optimal connection relations with one FPSO, $m$ cluster manifolds and l PLEMs. Our goal is to obtain the optimal layout scenario of cluster manifold at the lowest layout cost with the optimal number and locations of cluster manifolds and PLEMs.

If the coordinates of one FPSO, $m$ cluster manifolds and $l$ PLEMs are expressed by $M_{F P S O},\left(M_{M 1}, M_{M 2}, \cdots, M_{M m}\right)$, and $\left(M_{P M 1}, M_{P M 2}, \cdots M_{P M l}\right)$, respectively, the mathematical model of the layout of cluster manifold can be roughly translated into the following optimization problem:

$\min _{M_{F P S O}, M_{M 1}, \cdots, M_{M m}, M_{P M 1}, \cdots M_{P M L}, m, l} C\left(M_{F P S O}, M_{M 1}, \cdots, M_{M m}, M_{P M 1}, \cdots M_{P M L}, m, l\right)$

Here, $C\left(M_{F P S O}, M_{M 1}, \cdots, M_{M m}, M_{P M 1}, \cdots M_{P M L}, m, l\right)$ is the layout cost function based on the connection constraint conditions among FPSO ,cluster manifolds and PLEMs. The problem (1) is an implicit expression, which is a discrete optimization problem. In addition, the locations and number of cluster manifolds and PLEMs are not known. The number of well slots on the cluster manifold will be changed along with the value of $m$. Meanwhile, there are some special constraint conditions of the cluster manifold layout. Hence, the process of solving problem (1) is complicated.

To solve the problem (1), three steps of local optimization need to be conducted, which are partition of subsea wells, the connection paths without PLEMs, and the optimization layout with the PLEMs. Next, we will analyze the difficulties and challenges in each optimization problem.

\section{A. Locations of the Cluster Manifolds}

In real engineering, it is the connection facility that is used to connect any two subsea production facilities among subsea trees, cluster manifolds, PLEMs or risers. Generally speaking, connection facilities in deepwater mainly include jumpers, connectors, pipeline end terminations (PLETs), in-line tees, in-line sleds and branch flowlines. The length of jumpers should not be too short because it is used to release the HT/HP oil and gas and thermal stress, which needs certain flexibility. Meanwhile, the length of jumpers should also not be too long because it may cause vortex-induced vibration and torsion deformation due to self-weight and environmental loads. Therefore, the length of jumpers is usually at $15 \mathrm{~m} \sim 100 \mathrm{~m}$, in which $20 \mathrm{~m} \sim 60 \mathrm{~m}$ is more common. If the distance between two subsea facilities is within this range, it can be connected directly by jumpers. Otherwise, other connection facilities may be required such as PLETs, branch flowlines or in-line tees. Different connection types between subsea facilities may make a huge impact on the cost, risk and pigging of the layout scenarios.

Hence, if we want to find the optimal solution of problem (1) in real engineering, different connection types between subsea facilities need to be considered in the mathematical model. Wang et al. (2012) presented a mathematical model for the partition of subsea wells in the layout of cluster manifold. Some simulations were performed to prove the validity of the model and its algorithm. The results showed that it was a good attempt to obtain the optimal layout scenario by the mathematical model, which can provide a quantitative reference to the engineers for making their important decisions. The locations and number of cluster manifolds, the number of well slots, the minimal cost and the optimal layout can be obtained when the number and locations of subsea wells were known.

In their work, they assumed that $n$ subsea wells $p_{1}, p_{2}, \cdots, p_{n}$ were to be partitioned into $m$ cluster manifolds $k_{1}, k_{2}, \cdots, k_{m} \quad$. By the connection matrix $X=\left(x_{i j}\right)_{m \times n} \in\{0,1\}$ and the cost matrix $C=\left(c_{i j}\right)_{m \times n}$ between subsea wells and cluster manifolds, the mathematical model for the partition of subsea wells can be yielded by solving the following optimization problem:

$$
\mathrm{P} 1: \quad \min _{C, X} f(C, X)
$$

where $f(C, X)=\left(\begin{array}{c}c_{11} X_{11}+c_{12} X_{12}+\cdots+c_{1 n} x_{1 n} \\ +c_{21} x_{21}+c_{22} x_{22}+\cdots+c_{2 n} x_{2 n} \\ +, \cdots,+ \\ +c_{m 1} x_{m 1}+c_{m 2} x_{m 2}+, \cdots,+c_{m n} x_{m n}\end{array}\right)$ and the square of the Euclidean distance between the subsea wells $p_{j}$ and the cluster manifolds $k_{i}$ is denoted by $c_{i j}=\left\|p_{j}-M_{i}\right\|^{2}$ $i=1,2, \cdots m, j=1,2, \cdots, n$.

Thus, the initial optimal solution of this partition problem can be given if the connection and cost matrix were known. The connection matrix can be easily gotten by any given connection relations between subsea wells and cluster manifolds. But the cost matrix may be obtained after the distances between subsea wells and cluster manifolds were determined. Therefore, the locations of cluster manifolds were crucial in the model. Due to the complexity of the partition problem, in this model, the connection types between subsea wells and cluster manifolds were simplified into straight pipelines, not including the other subsea connection facilities, such as jumpers, PLETs and so on. In other words, there are just line segments between subsea wells and cluster manifolds. As a result, the layout costs of subsea well $p_{i_{j}}\left(x_{i}, y_{j}\right)$ connected to cluster manifold $k_{i}\left(x_{M_{i}}, y_{M_{i}}\right)$ were simplified by the square of the Euclidean distance, i.e., 


$$
\left\|p_{i_{j}}-M_{i}\right\|^{2}=\left(x_{j}-x_{M_{i}}\right)^{2}+\left(y_{j}-y_{M_{i}}\right)^{2} \quad i, j=1,2, \cdots, k
$$

The locations of cluster manifolds can be optimized by solving the optimization problem in equation (4), which is a simple unconstrained optimization problem.

$$
\begin{aligned}
\min g(x, y)= & \min \sum_{j=1}^{k}\left\|p_{i_{j}}-M_{i}\right\|^{2} \\
= & \min \left[\left(x-x_{1}\right)^{2}+\left(y-y_{1}\right)^{2}+\cdots+\right. \\
& \left.\left(x-x_{k}\right)^{2}+\left(y-y_{k}\right)^{2}\right]
\end{aligned}
$$

By calculating, a solution to the ideal location of cluster manifold in a well set was the arithmetic mean of the coordinates of subsea wells connected to this cluster manifold. Consequently, the cost matrix was obtained based on the locations of cluster manifolds. In the algorithm, the sum of layout cost between each well and the cluster manifold in the same well set could be reduced by adjusting the relation and cost matrix, continually. Possible optimal partition was gotten (See Fig. 3) until the locations of cluster manifolds did not be changed.

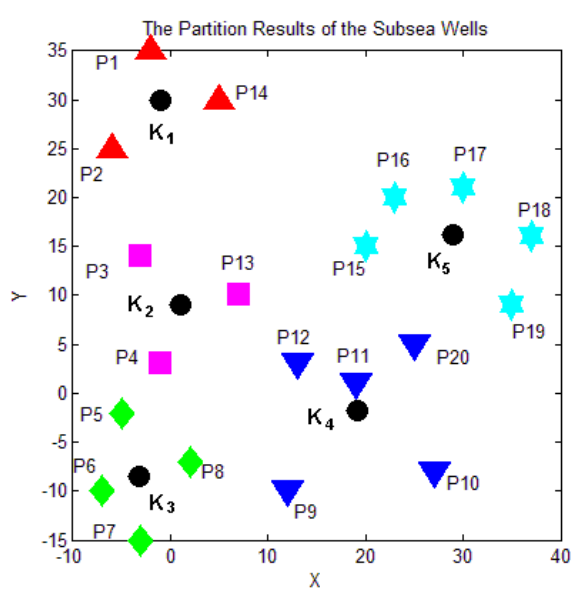

\section{FIGURE III. PARTITIONS OF FIVE CLUSTER MANIFOLDS (M=5)}

However, the results of optimal partition were the approximations of ideal solutions, which were not applied to the real project because the connection facilities were not included. If two connection types between subsea well $p_{j}$ and cluster manifold $k_{i}$ are considered, the definition of layout cost will become more complicated, rather than the square of the Euclidean distance of pipelines, which may increase the difficulty to get the solution of the locations of cluster manifolds. Hence, the connection types by the jumpers or jumpers, PLETs and infield flowlines are one of the challenges in the model, which shall be studied further.

\section{B. Optimal Connection Paths without the PLEMs}

When the number and locations of cluster manifolds, and the location of floating production platform are available, the connection paths between cluster manifolds and FPSO need to be found. Fig. 4 shows several possible connection paths in the layout of cluster manifolds, where there are 4 cluster manifolds and 1 FPSO. Our problem is to find which one is the optimal layout at the lowest cost. Here, the PLEM is not considered now.

In the layout of cluster manifold, there are some characteristics as follows in Fig.4.

B1: The series connection between cluster manifolds is shown in the same production loops.

B2: Any two pipelines can not be allowed to cross each other in any production loop.

B3: The whole loops in the layout scenarios are open and no closed loops can be formed.

B4: All of the cluster manifolds are connected to the FPSO, which can form one or more production loops.

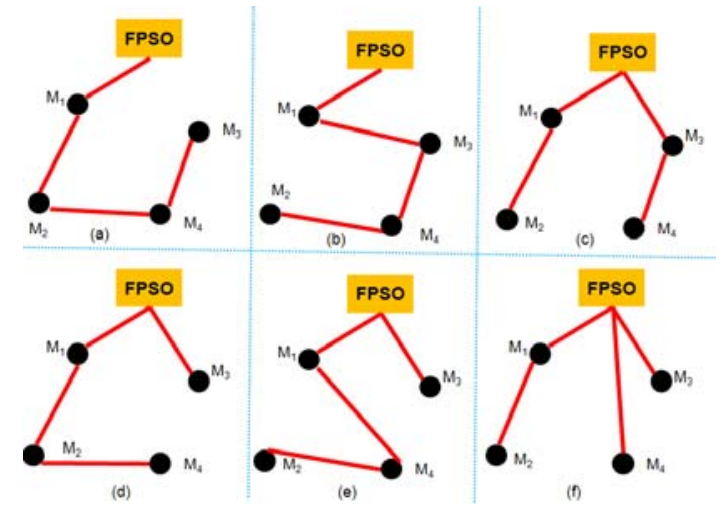

FIGURE IV. POSSIBLE CONNECTION PATHS

Those are also four main challenges, which shall be handled as the constraint conditions. If 1 FPSO and $m$ cluster manifolds $M_{1}, M_{2}, \cdots M_{m}$ can be defined by $N_{i}(x, y), i=1,2, \cdots,(m+1)$, connection relations and cost matrix between FPSO and cluster manifolds can be given as $X=\left(x_{i j}\right)_{(m+1) \times(m+1)} \in\{0,1\}$ and $C=\left(c_{i j}\right)_{(m+1) \times(m+1)}$, respectively. Thus, our target function can be obtained as follows:

$$
\mathrm{P} 2: \min _{X, C, N_{1}, N_{2}, \cdots, N_{(m+1)}} f(C, X)=\min _{X, C, N_{1}, N_{2}, \cdots, N_{(m+1)}} \frac{1}{2} \sum_{i, j} x_{i j} C_{i j}
$$

where $N_{i}(x, y)=M_{F 1}(x, y), M_{M 1}(x, y), \cdots M_{M m}(x, y)$.

In fact, if we take the FPSO as the root and the cluster manifolds as the nodes, the layout of cluster manifolds can be regarded as a special tree without leaves in Fig.4. This optimization problem looks like the minimum spanning tree (MST) problem for a weighted graph, which is to find a spanning tree of the graph with weight that is less than or equal 
to the weight of every other spanning tree (Fredman and Willard,1994). But there is a great difference between the P2 and the MST. In the P2, the degree of each cluster manifold is all 1 or 2 , which ensures that cluster manifolds can be connected in series. Specifically, if $\operatorname{deg}(\mathrm{i})$ is denoted as the degree of the node, we can have

$$
\operatorname{deg}(i)=\left\{\begin{array}{c}
1,2, \cdots, m \quad i=1 \\
1,2 \quad i=2, \cdots,(m+1)
\end{array}\right.
$$

But in the MST, the degree of each mode can be 1,2 , or 3 , i.e., the subsea wells are not constrained to be connected in series. This is one main difference between MST and P1. In addition, there is no less than one production loop connected to FPSO, therefore, the degree of FPSO is optimal. In the MST, the degree of the FPSO is a fixed value. Moreover, it is obvious that this optimization is not the travelling salesman problem (TSP) because the degree of each node does not need to be 2 and the optimal layout loop is open (Dorigo and Gambardella, 1997).

Thus, the optimization layout of cluster manifold is a special non line programming problem, which is different from the MST and TSP. Considering the challenges of B1 B4 in the $\mathrm{P} 2$, the implementation of algorithm for solving this optimization problem is quite complicated.

\section{The Number and Locations of PLEMs}

The PLEM is a key subsea facility, which can be applied to optimize the layout scenario further by reducing the number of flowlines when there are two or more cluster manifolds. If current optimal connection relations are known, our problem is to find the number and locations of PLEMs which can reduce the layout cost. Possible layout scenarios are shown in Fig. 5(Wang et al, 2012 and Damsleth et al, 2012).

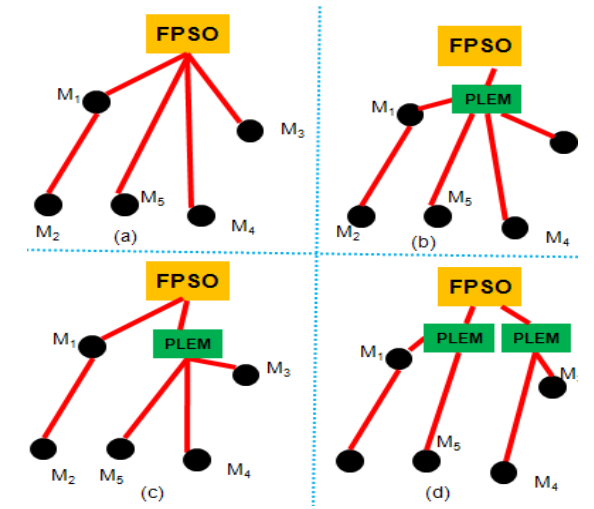

FIGURE V. POSSIBLE LAYOUT SCENARIOS WITH THE PLEM

Based on the characters of B1 B4 in the connection paths, the connection relations among cluster manifolds, PLEM and FPSO are constrained. In Fig. 5, no less than two cluster manifolds are connected to the PLEM whose one end must be connected to the FPSO. If 1 FPSO, $m$ cluster manifolds $M_{1}, M_{2}, \cdots M_{m}$ and $l$ PLEMs $P M_{1}, P M_{2}, \cdots P M_{l}$ can be defined by $N_{i}(x, y)$, $i=1,2, \cdots,(m+1),(m+2), \cdots,(m+1+l)$.

Here, FPSO is taken as the first point. Based on the connection relation and cost matrix, the mathematical model for the optimization of subsea wells in the layout of cluster manifold with the PLEMs can be given as follows:

$$
\begin{gathered}
\text { P3: } \min _{X, C, N_{1}, N_{2}, \cdots, N_{(m+1)}, \cdots, N_{(m+1+l)}, m, l}\left[f(C, X)+\sum_{k=1}^{l} C_{k}\right] \\
\text { where } \quad X=\left(x_{i j}\right)_{(m+1+l) \times(m+1+l)} \in\{0,1\} \quad, \quad C=\left(c_{i j}\right)_{(m+1+l) \times(m+1+l)}
\end{gathered}
$$
and $C_{k}$ is the cost of $k$-th PLEM, including the design, manufacture and installation.

The degree of PLEM is the number of edges that can be connected to it, which can be decided by the number of cluster manifolds and connection relations. If the cluster manifolds connected to the PLEM are known, the location of this PLEM can be gotten according to the solution to the P1.Similiarly, if the connection facilities are included in this model, the algorithm will become more difficult based on the conditions of B1 B4.

\section{CONCLUDING REMARKS}

In this paper, the optimization of the layout of cluster manifold is discussed by a new mathematical model concept. The mathematical models in relation to the partition for subsea wells and connection paths among cluster manifolds, FPSO and PLEMs are presented and the challenges are analyzed for the next implementation of algorithms. The research on the layout of cluster manifold by the mathematical model is a new validity attempt, which can provide a quantitative reference tool to the engineers and accelerate the evaluation process of the optimal layout scenario.

\section{ACKNOWLEDGMENTS}

The authors are grateful for the financial support by China National Science and Technology Major Project "Virtual Simulation Systems in Ocean Engineering” (No. 2016ZX05033-004-003), National Key Research and Development Plan (No. 2016YFC0303701) and Science Foundation of China University of Petroleum-Beijing (No. 2462014YJRC032).

\section{REFERENCES}

[1] Ciaurri D.E., Conn A.R., Mello U.T., et al. Integrating mathematical optimization and decision making in intelligent fields[C]. In: SPE Intelligent Energy International, 27-29 March 2012, Utrecht, The Netherlands, SPE149780.

[2] Damsleth, P., Abdalla, B., Tang K.C, 2012. Challenges and concept solutions for 46-inch diameter deepwater PLEM - connector qualification [C]. In: Proceedings of the Twenty-second International Offshore and Polar Engineering Conference, Rhodes, Greece.

[3] Dorigo M. and Gambardella L.M. Ant Colony System : a cooperative learning approach to the traveling salesman problem [J]. IEEE Transactions on Evolutionary Computation, 2007, Volume 1,No.1, pp. 53-66.

[4] Fredman, M.L. and Willard, D.E. Trans-dichotomous algorithms for minimum spanning trees and shortest paths [J]. Journal of Computer and System Sciences, 1994, 48 (3): 533-551. 
[5] Kaiser M.J. and Allan G. Generalized Functional Models for Drilling Cost Estimation [J]. SPE Drilling\&Completion, 2007,Vol 22(2):67-73.

[6] Mclnturff T.L. Troika subsea production template and manifold system [C]. Offshore Technology Conference, Houston, Texas, USA, 4-7 May 1998, OTC8847.

[7] Nelson S G. AKPO: The Subsea Production System[C], Offshore Technology Conference, Houston, Texas, USA, 4-7 May, 2010, OTC20933.

[8] Onwunalu J.E. and Durlofsky L.J. Development and application of a new well pattern optimization algorithm for optimizing large scale field development [C]. In: SPE Annual Technical Conference and Exhibition, 4-7 October 2009, New Orleans, Louisiana,SPE 124364.

[9] Stock, P.F.K, Kuppens, M.L., Silva, J.L.B., et al. Roncador Manifolds Installation by the Pendulous Method [C]. In: Proceedings of the 2007 Deep Offshore Technology, Stavanger, Norway.

[10] Underwood N, Odina L, Hansen K, Lassila H. Integrated Subsea Design: An Informed and Practical Approach [C]. In: ASME 27th International Conference on Offshore Mechanics and Arctic Engineering, Estoril, Portugal,OMAE2008-57207,Vol. 3, pp.175-182,2008.

[11] Wang Y.Y., Duan M.L., Feng W., et al. An exploratory study on the optimum layout of the cluster manifold [C]. In: Proceedings of 31nd International Conference on Offshore Mechanics and Arctic Engineering, OMAE2012, Rio, Brazil, 30, June-6, July.

[12] Wang Y.Y., Duan M.L., Xu M.H., et al. A mathematical model for subsea wells partition in the layout of cluster manifolds [J]. Applied Ocean research, June 2012,Vol 36, pp.26-35. 